MMCA-97 Conference, pp. 143-151

R. Čiegis (Ed)

(C) 1997 Vilniaus Gedimino technikos universitetas

\title{
ON ASYMPTOTICS OF A POPULATION MODEL WITH RANDOM MATING
}

\author{
VLADAS SKAKAUSKAS \\ Faculty of Mathematics, Vilnius University, \\ Naugarduko 24, Vilnius 2006, Lithuania \\ E-mail: vladas.skakauskas@maf.vu.lt
}

\begin{abstract}
This paper deals with a model for an age-sex structured population consisting of male, single and fertilized female subclasses taking into account a random coupling of sexes (for a period of mating only) and females' pregnancy. For certain forms of the demographic rates there are presented separable solutions, and the asymptotic behaviour of the general solution is demonstrated.
\end{abstract}

\section{INTRODUCTION}

In the recent papers [2], [3] we introduced a model for an age-sex-stuctured population consisting of male, single and fertilized female subclasses taking into account a random coupling of sexes (for a period of mating only), and pregnancy of females.

Let us first recall the following notions in [3]:

$\tau_{1}, \tau_{2}$ and $\tau_{3}$ denote the ages of males, females and embryos, respectively, $t$ is time,

$u_{1}\left(t, \tau_{1}\right), u_{2}\left(t, \tau_{2}\right)$ and $u_{3}\left(t, \tau_{1}, \tau_{2}, \tau_{3}\right)$ are age densities of numbers of males, single and fertilized females, respectively;

$p\left(t, \tau_{1}, \tau_{2}\right)$ is the females' fertilization rate;

$\nu_{1}\left(t, \tau_{1}\right), \nu_{2}\left(t, \tau_{2}\right)$ and $\nu_{3}\left(t, \tau_{1}, \tau_{2}, \tau_{3}\right)$ are death rates of males, single and fertilized females, respectively;

$X_{2}\left(u_{3}\right)\left(t, \tau_{2}\right)$ gives the females' supply rate due to conceiving and deliveries;

$\sigma_{1}=\left(\tau_{11}, \tau_{12}\right], 0<\tau_{11}<\tau_{12}<\infty$ is the males' sexual activity interval, $\bar{\sigma}_{1}=\left[\tau_{11}, \tau_{12}\right]$

$\sigma_{3}=(0, T], 0<T<\infty$ is the females' gestation interval, $\bar{\sigma}_{3}=[0, T] ;$ 
$\sigma_{2}\left(\tau_{3}\right)=\left(\tau_{21}+\tau_{3}, \tau_{22}+\tau_{3}\right], 0<\tau_{21}<\tau_{22}<\infty, \bar{\sigma}_{2}\left(\tau_{3}\right)=\left[\tau_{21}+\tau_{3}, \tau_{22}+\tau_{3}\right], ;$

$\sigma_{2}(0)$ and $\sigma_{2}(T)$ are the females' fertilization and reproductivity intervals, respectively;

$n_{1}(t)$ is the size of males' subclass with ages from $\sigma_{1}$;

$b_{1}\left(t, \tau_{1}, \tau_{2}\right)$ and $b_{2}\left(t, \tau_{1}, \tau_{2}\right)$ represent the expectied numbers of offspring produced at time $t$ by a fertilized female of characteristics $\left(\tau_{1}, \tau_{2}, T\right)$ and having the sex of males and females, respectively;

$u_{1}^{0}\left(\tau_{1}\right), u_{2}^{0}\left(\tau_{2}\right), u_{3}^{0}\left(\tau_{1}, \tau_{2}, \tau_{3}\right)$ are the initial distributions;

$\sigma=\sigma_{1} \times \sigma_{2}(T), d \sigma=d \tau_{1} d \tau_{2}$

$\tau_{2}^{0}=0, \tau_{2}^{1}=\tau_{21}, \tau_{2}^{2}=\min \left(\tau_{21}+T, \tau_{22}\right), \tau_{2}^{3}=\max \left(\tau_{21}+T, \tau_{22}\right), \tau_{2}^{4}=$ $\tau_{22}+T, \tau_{2}^{5}=\infty$

$I=(0, \infty), \bar{I}=[0, \infty], I_{4}=\left(\tau_{2}^{4}, \infty\right), I_{s}=\left(\tau_{2}^{s}, \tau_{2}^{s+1}\right], s=\overline{0,3}$

$Q_{1}=I \times I, Q_{2}=I \times\left(I \backslash \bigcup_{s=1}^{4} \tau_{2}^{s}\right), Q_{3}=I \times \sigma_{1} \times \sigma_{2}\left(\tau_{3}\right) \times \sigma_{3} ;$

$\left[\left.u_{2}\right|_{\tau_{2}=\tau_{2}^{s}}\right]$ is a jump of $u_{2}$ at the plane $\tau_{2}=\tau_{2}^{s}$;

$L_{j}=\partial / \partial t+\partial / \partial \tau_{j}, j=1,2, L_{3}=L_{2}+\partial / \partial \tau_{3}, X_{1}\left(u_{3}\right)=X_{3}\left(u_{3}\right)=0$,

$D_{1}=2^{1 / 2} \tilde{D}_{1}, 2^{1 / 2} \tilde{D}_{2}, D_{3}=3^{1 / 2} \tilde{D}_{3}$, where $\tilde{D}_{i}, i=1,2,3$ is the directional derivative along the positive direction of characteristics of the operator $L_{i}$;

$C(\Omega), C^{1}(\Omega)$ and $L^{1}(\Omega)$ denote spaces of continuous, continuously differentiable and absolutely integrable functions on $\Omega$, respectively.

Note that, if the partial derivatives with respect to $t$ and $\tau_{j}$ exist, then $D_{i} u_{i}=L_{i} u_{i}, i, j=1,2,3$.

The system

$D_{i} u_{i}+\nu_{i} u_{i}-X_{i}\left(u_{3}\right)=0$ in $Q_{i}, i=1,2,3$,

$X_{2}\left(u_{3}\right)=-\left\{\begin{array}{l}0, \tau_{2} \notin \sigma_{2}(0) \\ \left.\int_{\sigma_{1}} u_{3}\right|_{\tau_{3}=0} d \tau_{1}, \tau_{2} \in \sigma_{2}(0)\end{array}+\left\{\begin{array}{l}0, \tau_{2} \notin \sigma_{2}(T) \\ \left.\int_{\sigma_{1}} u_{3}\right|_{\tau_{3}=T} d \tau_{1}, \tau_{2} \in \sigma_{2}(T)\end{array}\right.\right.$

supplemented by the conditions

$$
\begin{gathered}
\left.u_{j}\right|_{\tau_{j}=0}=\left.\int_{\sigma} b_{j} u_{3}\right|_{\tau_{3}=T} d \sigma, j=1,2,\left[\left.u_{2}\right|_{\tau_{2}=\tau_{2}^{s}}\right]=0, s=\overline{1,4}, \\
\left.u_{3}\right|_{\tau_{3}=0}=p u_{1} u_{2} / n_{1}, n_{1}=\int_{\sigma_{1}} u_{1} d \tau_{1}
\end{gathered}
$$




$$
\left.u_{i}\right|_{t=0}=u_{i}^{0}, i=1,2,3,
$$

governs the evolution of the population (see[2]). Here the mating function $p u_{1} u_{2} / n_{1}$ represents the age density of females conceiving on the period of random mating. Non-negative functions $\nu_{i}, b_{j}, p, u_{j}^{0}$ are assumed to be prescribed. In addition we assume that initial functions $u_{1}^{0}, u_{2}^{0}, u_{3}^{0}$ must satisfy the following compatibility conditions

$$
\begin{gathered}
u_{j}^{0}=\left.\left.\int_{\sigma} b_{j}\right|_{t=0} u_{3}^{0}\right|_{\tau_{3}=0} d \sigma, j=1,2,\left[\left.u_{2}^{0}\right|_{\tau_{2}=\tau_{2}^{s}}\right]=0, s=\overline{1,4}, \\
\left.u_{3}^{0}\right|_{\tau_{3}=0}=\left.p\right|_{t=0} u_{1}^{0} u_{2}^{0} / \int_{\sigma_{1}} u_{1}^{0} d \tau_{1} .
\end{gathered}
$$

As it follows from the biological meaning the unknown functions $u_{1}, u_{2}, u_{3}$ also must be non-negative. The unique solution of (1)-(4) problem has been constructed by Skakauskas [2].

In the present work we limit our attention to the case where $p, b_{1}, b_{2}, \nu_{3}$ do not depend of $\tau_{1}$ and $t$. This paper consists of two sections. In the first of them we obtain the product solutions of (1), (2), while in the other one the asymptotic behaviour of the general solution of (1)-(4) is demonstrated. We consider the case of multiple deliveries, i.e. $\tau_{22}-\tau_{21}>T\left(\tau_{2}^{2}=\tau_{21}+T, \tau_{2}^{3}=\right.$ $\left.\tau_{22}\right)$. All results obtained in this paper can be applied for the opposite case.

\section{PRODUCT SOLUTIONS OF (1)-(2)}

In this section a particular solution of (1)-(2) will be considered. Assume $D=2^{1 / 2} \tilde{D}$, where $\tilde{D}$ is the directional derivative along the characteristics of the operator $\partial / \partial \tau_{2}+\partial / \partial \tau_{3}$, and let

$$
\tilde{\nu}_{2}\left(\tau_{2}\right)=\left\{\begin{array}{l}
o, \tau_{2} \notin \sigma_{2}(0) \\
p, \tau_{2} \in \sigma_{2}(0) .
\end{array}\right.
$$

Substituting

$$
\begin{gathered}
u_{1}=c_{1}(\lambda) c_{2} f_{1}\left(\tau_{1}\right) \exp \left\{\lambda\left(t-\tau_{1}\right)\right\}, f_{1}(0)=1, \\
u_{2}=c_{2} f_{2}\left(\tau_{2}\right) \exp \left\{\lambda\left(t-\tau_{2}\right)\right\}, f_{2}(0)=1, \\
u_{3}=c_{2} f_{3}\left(\tau_{2}, \tau_{3}\right) f_{4}\left(\lambda, \tau_{1}\right) \exp \left\{\lambda\left(t-\tau_{2}\right)\right\}, \\
f_{4}\left(\lambda, \tau_{1}\right)=f_{1}\left(\tau_{1}\right) \exp \left\{-\lambda \tau_{1}\right\} / \int_{\sigma_{1}} f_{1}(\xi) \exp \{-\lambda \xi\} d \xi,
\end{gathered}
$$


where $\lambda, c_{1}(\lambda), c_{2}$ are some constants, into (1), (2) we arrive at the system

$$
\begin{aligned}
& d f_{1} / d \tau_{1}=-\nu_{1} f_{1}, f_{1}(0)=1, \\
& D f_{3}=-\nu_{3} f_{3}, f_{3}\left(\tau_{2}, 0\right)=p\left(\tau_{2}\right) f_{2}\left(\tau_{2}\right), \\
& d f_{2} / d \tau_{2}=-\tilde{\nu}_{2} f_{2}+\left\{\begin{array}{l}
0, \tau_{2} \notin \sigma_{2}(T), f_{2}(0)=1, \\
f_{3}\left(\tau_{2}, T\right), \tau_{2} \in \sigma_{2}(T),\left[f_{2}\left(\tau_{2}^{i}\right)\right]=0, i=\overline{1,4},
\end{array}\right. \\
& c_{1}=\int_{\sigma_{2}(T)} F_{1}\left(\tau_{2}\right) \exp \left\{-\lambda \tau_{2}\right\} d \tau_{2}, 1=\int_{\sigma_{2}(T)} F_{2}\left(\tau_{2}\right) \exp \left\{-\lambda \tau_{2}\right\} d \tau_{2} ;
\end{aligned}
$$

here $F_{i}\left(\tau_{2}\right)=b_{i}\left(\tau_{2}\right) f_{3}\left(\tau_{2}, T\right), i=1,2$. Thus

$$
f_{1}=\exp \left\{-\int_{0}^{\tau_{1}} \nu_{1}(\xi) d \xi\right\}, f_{3}=\left.\left(p f_{2}\right)\right|_{\tau_{2}-\tau_{3}} \exp \left\{-\int_{0}^{\tau_{3}} \nu_{3}\left(\xi+\tau_{2}-\tau_{3}, \xi\right) d \xi\right\}
$$

and

$$
\begin{aligned}
& d f_{2} / d \tau_{2}=-\tilde{\nu}_{2} f_{2}+\left\{\begin{array}{l}
0, \tau_{2} \notin \sigma_{2}(T), f_{2}(0)=1, \\
q\left(\tau_{2}\right) f_{2}\left(\tau_{2}-T\right), \tau_{2} \in \sigma_{2}(T),\left[f_{2}\left(\tau_{2}^{i}\right)\right]=0, i=\overline{1,4},
\end{array}\right. \\
& q=p\left(\tau_{2}-T\right) \exp \left\{-\int_{0}^{T} \nu_{3}\left(\xi+\tau_{2}-T, \xi\right) d \xi\right\} .
\end{aligned}
$$

Due to the delay argument the unique solution of (8) can be easily constructed. Then, from characteristic equation $(6)_{2}$ we define $\lambda$, and then from $(6)_{1}$ one can obtain $c_{1}(\lambda), c_{2}>0$ is arbitrary. It is well known [1] that roots $\lambda_{k}=\alpha_{k} \pm i \beta_{k}, i=\sqrt{-1}$ of $(6)_{2}$ are such that $\beta_{0}=0$, sign $\alpha_{0}=$ $\operatorname{sign}\left\{\int_{\sigma_{2}(\mathrm{~T})} \mathrm{b}_{2}\left(\tau_{2}\right) \mathrm{f}_{3}\left(\tau_{2}, \mathrm{~T}\right) \mathrm{d} \tau_{2}-1\right\}, \alpha_{\mathrm{k}}<\alpha_{0}$ for $k=1,2, \ldots$ provided $b_{2} f_{3} \in$ $L^{1}\left(\sigma_{2}(T)\right)$. (2).

We call the solution of type (5) the product (or separable) solution of (1)-

THEOREM 1. Given $c_{2}>0$ and the non-negative functions $p \in C\left(\bar{\sigma}_{2}(0)\right), \nu_{1}$, $\nu_{2} \in C(\bar{I}), \nu_{3} \in C\left(\bar{\sigma}_{2}\left(\tau_{3}\right) \times \bar{\sigma}_{3}\right), b_{j} \in L_{1}\left(\sigma_{2}(T)\right)$. Then (1)-(2) admits the non-negative product solution (5).

\section{ASYMPTOTIC BEHAVIOUR OF GENERAL SOLUTION OF (1)-(4)}

In this section we will obtain the asymptotic formula of the general solution for (1)-(4) problem. Let us denote 


$$
z\left(t, \tau_{2}, \tau_{3}\right)=\int_{\sigma_{1}} u_{3} d \tau_{1}, z^{0}\left(\tau_{2}, \tau_{3}\right)=\int_{\sigma_{1}} u_{3}^{0} d \tau_{1}, \tilde{\nu}_{2}\left(\tau_{2}\right)=\nu_{2}+p
$$

and formulate the following hypotheses:

$\left(H_{1}\right) p, \nu_{1}, \nu_{2}, \nu_{3}$ satisfy the conditions of Th.1, and $b_{i} \in C\left(\bar{\sigma}_{2}(T)\right), u_{1}^{0}$,

$u_{2}^{0} \in C(\bar{I}) \cap L^{1}(I), u_{3} \in C\left(\bar{\sigma}_{1} \times \bar{\sigma}_{2}\left(\tau_{3}\right) \times \bar{\sigma}_{3}\right)$,

$$
\begin{aligned}
& \left(H_{2}\right) u^{*}=\sup _{I} u_{2}^{0}, z^{0 *}=\sup _{\sigma_{2}\left(\tau_{3}\right) \times \sigma_{3}} z^{0}, \nu_{*}=\inf _{I} \nu_{2}, p^{*}=\sup _{\sigma_{2}(0)} p \\
& b=\int_{\sigma_{2}(T)} b_{2} d \tau_{2}, \alpha=\max \left(b p^{*}, 1, p^{*} / \nu_{*}\right), q=\max \left(b z^{0 *} / u^{*}, 1, z^{0 *} / u^{*} \nu_{*}\right)
\end{aligned}
$$

are finite positive constants.

Denoting $J=[0, \min (\mathrm{t}, \mathrm{T})]$ and solving (1) along the respective characteristics we obtain

$$
\begin{aligned}
& u_{3}=\left\{\begin{array}{l}
u_{3}^{0}\left(\tau_{1}, \tau_{2}-t, \tau_{3}-t\right) \exp \left\{-\int_{\tau_{3}-t}^{\tau_{3}} \nu_{3}\left(\xi+\tau_{2}-\tau_{3}, \xi\right) d \xi\right\}, t \in\left[0, \tau_{3}\right], \\
\left.\left\{p u_{1} u_{2} / n_{1}\right\}\right|_{\left(t-\tau_{3}, \tau_{1}, \tau_{2}-\tau_{3}\right)} \exp \left\{-\int_{0}^{\tau_{3}} \nu_{3}\left(\xi+\tau_{2}-\tau_{3}, \xi\right) d \xi\right\}, \tau_{3} \in J,
\end{array}\right. \\
& z=\left\{\begin{array}{l}
z^{0}\left(\tau_{2}-t, \tau_{3}-t\right) \exp \left\{-\int_{\tau_{3}-t}^{\tau_{3}} \nu_{3}\left(\xi+\tau_{2}-\tau_{3}, \xi\right) d \xi\right\}, 0 \leq t \leq \tau_{3}, \\
\left.\left(p u_{2}\right)\right|_{\left(t-\tau_{3}, \tau_{2}-\tau_{3}\right)} \exp \left\{-\int_{0}^{\tau_{3}} \nu_{3}\left(\xi+\tau_{2}-\tau_{3}, \xi\right) d \xi\right\}, \tau_{3} \in J,
\end{array}\right. \\
& u_{1}=\left\{\begin{array}{l}
u_{1}^{0}\left(\tau_{1}-t\right) \exp \left\{-\int_{\tau_{1}-t}^{\tau_{1}} \nu_{1}(\xi) d \xi\right\}, 0 \leq t \leq \tau_{1} \\
u_{1}\left(t-\tau_{1}, 0\right) \exp \left\{-\int_{0}^{\tau_{1}} \nu_{1}(\xi) d \xi\right\}, \tau_{1} \in[0, t]
\end{array}\right. \\
& u_{2}=\left\{\begin{array}{l}
u_{2}^{0}\left(\tau_{2}-t\right) \exp \left\{-\int_{\tau_{2}-t}^{\tau_{2}} \nu_{2}(\xi) d \xi\right\}, 0 \leq t \leq \tau_{2}, \tau_{2} \in I_{0}, \\
u_{2}\left(t-\tau_{2}, 0\right) \exp \left\{-\int_{0}^{\tau_{2}} \nu_{2}(\xi) d \xi\right\}, \tau_{2} \in[0, t], \tau_{2} \in I_{0},
\end{array}\right. \\
& u_{2}=\left\{\begin{array}{l}
u_{2}^{0}\left(\tau_{2}-t\right) \exp \left\{-\int_{\tau_{2}-t}^{\tau_{2}} \tilde{\nu}_{2}(\xi) d \xi\right\}, 0 \leq t \leq \tau_{2}-\tau_{2}^{1}, \tau_{2} \in I_{1}, \\
u_{2}\left(\tau_{2}^{1}+t-\tau_{2}, \tau_{2}^{1}\right) \exp \left\{-\int_{\tau_{2}^{1}}^{\tau_{2}} \tilde{\nu}_{2}(\xi) d \xi\right\}, t>\tau_{2}-\tau_{2}^{1}, \tau_{2} \in I_{1},
\end{array}\right.
\end{aligned}
$$




$$
\begin{aligned}
& u_{2}=\left\{\begin{array}{l}
u_{2}^{0}\left(\tau_{2}-t\right) \exp \left\{-\int_{\tau_{2}-t}^{\tau_{2}} \tilde{\nu}_{2}(\xi) d \xi\right\}+\int_{\tau_{2}-t}^{\tau_{2}} \exp \left\{-\int_{\eta}^{\tau_{2}} \tilde{\nu}_{2}(\xi) d \xi\right\} z(\eta+ \\
\left.t-\tau_{2}, \eta, T\right) d \eta, t \in\left[0, \tau_{2}-\tau_{2}^{2}\right], \tau_{2} \in I_{2}, \\
u_{2}\left(\tau_{2}^{2}+t-\tau_{2}, \tau_{2}^{2}\right) \exp \left\{-\int_{\tau_{2}^{2}}^{\tau_{2}} \tilde{\nu}_{2}(\xi) d \xi\right\}+ \\
\int_{\tau_{2}^{2}}^{\tau_{2}} \exp \left\{-\int_{\eta}^{\tau_{2}} \tilde{\nu}_{2}(\xi) d \xi\right\} z\left(\eta+t-\tau_{2}, \eta, T\right) d \eta, t>\tau_{2}-\tau_{2}^{2}, \tau_{2} \in I_{2},
\end{array}\right. \\
& u_{2}=\left\{\begin{array}{l}
u_{2}^{0}\left(\tau_{2}-t\right) \exp \left\{-\int_{\tau_{2}-t}^{\tau_{2}} \nu_{2}(\xi) d \xi\right\}+\int_{\tau_{2}-t}^{\tau_{2}} \exp \left\{-\int_{\eta}^{\tau_{2}} \nu_{2}(\xi) d \xi\right\} z(\eta+ \\
\left.t-\tau_{2}, \eta, T\right) d \eta, 0 \leq t \leq \tau_{2}-\tau_{2}^{3}, \tau_{2} \in I_{3}, \\
u_{2}\left(\tau_{2}^{3}+t-\tau_{2}, \tau_{2}^{3}\right) \exp \left\{-\int_{\tau_{2}^{3}}^{\tau_{2}} \nu_{2}(\xi) d \xi\right\}+ \\
\int_{\tau_{2}^{3}}^{\tau_{2}} \exp \left\{-\int_{\eta}^{\tau_{2}} \nu_{2}(\xi) d \xi\right\} z\left(\eta+t-\tau_{2}, \eta, T\right) d \eta, t>\tau_{2}-\tau_{2}^{3}, \tau_{2} \in I_{3},
\end{array}\right. \\
& u_{2}=\left\{\begin{array}{l}
u_{2}^{0}\left(\tau_{2}-t\right) \exp \left\{-\int_{\tau_{2}-t}^{\tau_{2}} \nu_{2}(\xi) d \xi\right\}, 0 \leq t \leq \tau_{2}-\tau_{2}^{4},, \tau_{2} \in I_{4}, \\
u_{2}\left(\tau_{2}^{4}+t-\tau_{2}, \tau_{2}^{4}\right) \exp \left\{-\int_{\tau_{2}^{4}}^{\tau_{2}} \nu_{2}(\xi) d \xi\right\}, t>\tau_{2}-\tau_{2}^{4}, \tau_{2} \in I_{4},
\end{array}\right. \\
& u_{j}(t, 0)=\int_{\sigma_{2}(T)} b_{j}\left(\tau_{2}\right) z\left(t, \tau_{2}, T\right) d \tau_{2}, j=1,2
\end{aligned}
$$

Then using $\left(H_{2}\right)$ from (10)-(17) we obtain the estimate $0 \leq u_{2} \leq u^{*} q \alpha^{k}$ for $t \in(k T,(k+1) T], k=0,1, \ldots$ or $0 \leq u_{2} \leq u^{*} q \alpha^{t / T}$ for $t>0$. The last estimate ensures the existence of the Laplace transform of $u$.

Let $\widehat{u}_{2}\left(\lambda, \tau_{2}\right)$ and $\widehat{z}\left(\lambda, \tau_{2}, \tau_{3}\right)$ be the Laplace transform of $u_{2}$ and $z$. Then from (10), (12)-(16) we obtain:

$$
\begin{gathered}
\widehat{z}=p\left(\tau_{2}-\tau_{3}\right) \widehat{u}_{2}\left(\lambda, \tau_{2}-\tau_{3}\right) \exp \left\{-\int_{0}^{\tau_{3}}\left(\lambda+\nu_{3}\left(\eta+\tau_{2}-\tau_{3}, \eta\right) d \eta\right\}+\right. \\
\int_{0}^{\tau_{3}} z^{0}\left(\xi+\tau_{2}-\tau_{3}, \xi\right) \exp \left\{-\int_{\xi}^{\tau_{3}}\left(\lambda+\nu_{3}\left(\eta+\tau_{2}-\tau_{3}, \eta\right)\right) d \eta\right\} d \xi,
\end{gathered}
$$




$$
\begin{aligned}
& \widehat{u}_{2}=\widehat{u}_{2}(\lambda, 0) \exp \left\{-\int_{0}^{\tau_{2}}\left(\lambda+\nu_{2}\right) d \eta\right\}+ \\
& \int_{0}^{\tau_{2}} u_{2}^{0}(\xi) \exp \left\{-\int_{\xi}^{\tau_{2}}\left(\lambda+\nu_{2}\right) d \eta\right\} d \xi, \tau_{2} \in I_{0} \\
& \widehat{u}_{2}=\widehat{u}_{2}\left(\lambda, \tau_{2}^{1}\right) \exp \left\{-\int_{\tau_{2}^{1}}^{\tau_{2}}(\lambda+\tilde{\nu}) d \eta\right\}+ \\
& \int_{\tau_{2}^{1}}^{\tau_{2}} u_{2}^{0}(\xi) \exp \left\{-\int_{\xi}^{\tau_{2}}(\lambda+\tilde{\nu}) d \eta\right\} d \xi, \tau_{2} \in I_{1} \\
& \widehat{u}_{2}=\widehat{u}_{2}\left(\lambda, \tau_{2}^{2}\right) \exp \left\{-\int_{\tau_{2}^{2}}^{\tau_{2}}(\lambda+\tilde{\nu}(\eta)) d \eta\right\}+ \\
& \int_{\tau_{2}^{2}}^{\tau_{2}}\left\{u_{2}^{0}(\xi)+\widehat{z}(\lambda, \xi, T)\right\} \exp \left\{-\int_{\xi}^{\tau_{2}}(\lambda+\tilde{\nu}(\eta)) d \eta\right\} d \xi, \tau_{2} \in I_{2}, \\
& \widehat{u}_{2}=\widehat{u}_{2}\left(\lambda, \tau_{2}^{3}\right) \exp \left\{-\int_{\tau_{2}^{3}}^{\tau_{2}}\left(\lambda+\nu_{2}(\eta)\right) d \eta\right\}+ \\
& \int_{\tau_{2}^{3}}^{\tau_{2}}\left\{u_{2}^{0}(\xi)+\widehat{z}(\lambda, \xi, T)\right\} \exp \left\{-\int_{\xi}^{\tau_{2}}\left(\lambda+\nu_{2}(\eta)\right) d \eta\right\} d \xi, \tau_{2} \in I_{3}, \\
& \widehat{u}_{2}(\lambda, 0)=\int_{\sigma_{2}(T)} b_{2}\left(\tau_{2}\right) \widehat{z}\left(\lambda, \tau_{2}, T\right) d \tau_{2} .
\end{aligned}
$$

Denoting

$\widehat{u}_{2}\left(\lambda, \tau_{2}\right)=\widehat{u}_{2}(\lambda, 0) v_{1}\left(\lambda, \tau_{2}\right)+v_{2}\left(\lambda, \tau_{2}\right), g_{1}\left(\lambda, \tau_{2}-T\right)=p\left(\tau_{2}-T\right) \exp \left\{-\int_{0}^{T}(\lambda+\right.$ $\left.\left.\nu_{3}\left(\eta+\tau_{2}-T, \eta\right)\right) d \eta\right\}, g_{2}\left(\lambda, \tau_{2}-T\right)=\int_{0}^{T} z^{0}\left(\xi+\tau_{2}-T, \xi\right) \exp \left\{-\int_{\xi}^{T}\left(\lambda+\nu_{3}(\eta+\right.\right.$ $\left.\left.\left.\tau_{2}-T, \eta\right)\right) d \eta\right\} d \xi$

from (18)-(23) we obtain

$$
\widehat{u}_{2}(\lambda, 0)=\psi(\lambda) /(1-\delta(\lambda))
$$




$$
\begin{gathered}
\psi(\lambda)=\int_{\sigma_{2}(0)} b(\xi+T)\left\{g_{1}(\lambda, \xi) v_{2}(\lambda, \xi)+g_{2}(\lambda, \xi)\right\} d \xi \\
\delta(\lambda)=\int_{\sigma_{2}(0)} b_{2}(\xi+T) g_{1}(\lambda, \xi) v_{1}(\lambda, \xi) d \xi
\end{gathered}
$$

where

$v_{1}\left(\lambda, \tau_{2}\right)=\exp \left\{-\int_{0}^{\tau_{2}}\left(\lambda+\nu_{2}\right) d \eta\right\}=v_{1}\left(0, \tau_{2}\right) \exp \left\{-\lambda \tau_{2}\right\}, \tau_{2} \in I_{0}$,

$v_{1}\left(\lambda, \tau_{2}\right)=v_{1}\left(\lambda, \tau_{2}^{1}\right) \exp \left\{-\int_{\tau_{2}^{1}}^{\tau_{2}}\left(\lambda+\tilde{\nu}_{2}\right) d \eta\right\}=v_{1}\left(0, \tau_{2}\right) \exp \left\{-\lambda \tau_{2}\right\}, \tau_{2} \in I_{1}$,

$v_{1}\left(\lambda, \tau_{2}\right)=v_{1}\left(\lambda, \tau_{2}^{2}\right) \exp \left\{-\int_{\tau_{2}^{2}}^{\tau_{2}}\left(\lambda+\tilde{\nu}_{2}\right) d \eta\right\}+\int_{\tau_{2}^{2}}^{\tau_{2}} \exp \left\{-\int_{\xi}^{\tau_{2}}\left(\lambda+\tilde{\nu}_{2}\right) d \eta\right\} g_{1}(\lambda, \xi-$ $T) v_{1}(\lambda, \xi-T) d \xi=v_{1}\left(0, \tau_{2}\right) \exp \left\{-\lambda \tau_{2}\right\}, \tau_{2} \in I_{2}$,

$v_{1}\left(\lambda, \tau_{2}\right)=v_{1}\left(\lambda, \tau_{2}^{3}\right) \exp \left\{-\int_{\tau_{2}^{3}}^{\tau_{2}}\left(\lambda+\nu_{2}\right) d \eta\right\}+\int_{\tau_{2}^{3}}^{\tau_{2}} \exp \left\{-\int_{\xi}^{\tau_{2}}\left(\lambda+\nu_{2}\right) d \eta\right\} g_{1}(\lambda, \xi-$ $T) v_{1}(\lambda, \xi-T) d \xi=v_{1}\left(0, \tau_{2}\right) \exp \left\{-\lambda \tau_{2}\right\}, \tau_{2} \in I_{3}$,

$v_{2}\left(\lambda, \tau_{2}\right)=\int_{0}^{\tau_{2}} u_{2}^{0}(\xi) \exp \left\{-\int_{\xi}^{\tau_{2}}\left(\lambda+\nu_{2}\right) d \eta\right\} d \xi, \tau_{2} \in I_{0}$,

$v_{2}\left(\lambda, \tau_{2}\right)=v_{2}\left(\lambda, \tau_{2}^{1}\right) \exp \left\{-\int_{\tau_{2}^{1}}^{\tau_{2}}\left(\lambda+\tilde{\nu}_{2}\right) d \eta\right\}+\int_{\tau_{2}^{1}}^{\tau_{2}} u_{2}^{0}(\xi) \exp \left\{-\int_{\xi}^{\tau_{2}}\left(\lambda+\tilde{\nu}_{2}\right) d \eta\right\} d \xi, \tau_{2} \in$ $I_{1}$

$v_{2}\left(\lambda, \tau_{2}\right)=v_{2}\left(\lambda, \tau_{2}^{2}\right) \exp \left\{-\int_{\tau_{2}^{2}}^{\tau_{2}}\left(\lambda+\tilde{\nu}_{2}\right) d \eta\right\}+\int_{\tau_{2}^{2}}^{\tau_{2}} \exp \left\{-\int_{\xi}^{\tau_{2}}\left(\lambda+\tilde{\nu}_{2}\right) d \eta\right\}\left\{u_{2}^{0}(\xi)+\right.$ $\left.g_{2}(\lambda, \xi-T)+v_{2}(\lambda, \xi-T) g_{1}(\lambda, \xi-T)\right\} d \xi, \tau_{2} \in I_{2}$,

$v_{2}\left(\lambda, \tau_{2}\right)=v_{2}\left(\lambda, \tau_{2}^{3}\right) \exp \left\{-\int_{\tau_{2}^{3}}^{\tau_{2}}\left(\lambda+\nu_{2}\right) d \eta\right\}+\int_{\tau_{2}^{2}}^{\tau_{2}} \exp \left\{-\int_{\xi}^{\tau_{2}}\left(\lambda+\nu_{2}\right) d \eta\right\}\left\{u_{2}^{0}(\xi)+\right.$ $\left.g_{2}(\lambda, \xi-T)+v_{2}(\lambda, \xi-T) g_{1}(\lambda, \xi-T)\right\} d \xi, \tau_{2} \in I_{3}$,

are known functions. Therefore

$\delta(\lambda)=\int_{\sigma_{2}(0)} b_{2}(\xi+T) g_{1}(0, \xi) v_{1}(0, \xi) \exp \{-\lambda \xi\} d \xi$.

Using the inverse Laplace transform we obtain $u_{2}(t, 0) \sim c_{2}\left(\lambda_{0}\right) \exp \left\{\lambda_{0} t\right\}$, $c_{2}\left(\lambda_{0}\right)=-\psi\left(\lambda_{0}\right) / d \delta /\left.d \lambda\right|_{\lambda=\lambda_{0}}>0$. Then from (9)-(17) we get the following asymptotic behaviour

$u_{1} \sim c_{2}\left(\lambda_{0}\right) c_{1}\left(\lambda_{0}\right) f_{1}\left(\tau_{1}\right) \exp \left\{\lambda_{0}\left(t-\tau_{1}\right)\right\}$,

$u_{2} \sim c_{2}\left(\lambda_{0}\right) f_{2}\left(\tau_{2}\right) \exp \left\{\lambda_{0}\left(t-\tau_{2}\right)\right\}$ 
$u_{3} \sim c_{2}\left(\lambda_{0}\right) f_{4}\left(\lambda_{0}, \tau_{1}\right) f_{3}\left(\tau_{2}, \tau_{3}\right) \exp \left\{\lambda_{0}\left(t-\tau_{2}\right)\right\}$

as $\max \left(\tau_{1}, \tau_{2}\right)<t, t \rightarrow \infty$, where $\lambda_{0}, c_{1}\left(\lambda_{0}\right), f_{1}, f_{2}, f_{3}, f_{4}$ are defined by $(6)_{2},(6)_{1},(7),(8)$.

Thus we can formulate the following

TheOrem 2. Assume the hypotheses $\left(H_{1}\right),\left(H_{2}\right)$ hold. Then (25) is asymptotics of the general solutuion for (1)-(4) as $\max \left(\tau_{1} \cdot \tau_{2}\right)<t, t \rightarrow \infty$.

\section{REFERENCES}

[1] A.A. Gimelfarb, L.R. Ginzburg, R.A. Poluektov, Ju. A. Pykh and V.A. Ratner, Theory of biological populations dynamics, (in Russian), Nauka, Moscow, 1974.

[2] V.Skakauskas, An evolution of an autosomal polylocal polyallelic diploid population taking into account crossing-over and gestation period, Lithuanian Mathematical Journal, 34 no 3 (1994), 288-299.

[3] V.Skakauskas, The unique solvability of a non-migrating limited panmiction population evolution problem, Informatica (Lithuanian Academy of sciences) 7 no 2: 229-254 (1996). 